\title{
Control of Leaky-Mode Propagation and Radiation Properties in Hybrid Dielectric-Waveguide Printed-Circuit Technology: Experimental Results
}

José Luis Gómez-Tornero, Member, IEEE, George Goussetis, Member, IEEE, Alexandros P. Feresidis, Member, IEEE, and Alejandro Alvarez Melcón, Member, IEEE

\begin{abstract}
Experimental results are presented to show how a planar circuit, printed on a laterally shielded dielectric waveguide, can induce and control the radiation from a leaky-mode. By studying the leaky-mode complex propagation constant, a desired radiation pattern can be synthesized, controlling the main radiation characteristics (pointing direction, beamwidth, sidelobes level) for a given frequency, This technique leads to very flexible and original leaky-wave antenna designs. The experiments show to be in very good agreement with the leaky-mode theory.
\end{abstract}

Index Terms-Hybrid waveguide printed circuit technology, leaky-wave antennas, sidelobes level control, tapering antennas.

\section{INTRODUCTION}

A NY radiating system or antenna must be capable for transmitting and for receiving electromagnetic signals in a certain frequency band, and with certain performance. A flexible antenna would be one able to control its radiation pattern characteristics without restrictions, while presenting good input matching for the desired frequency band. The antenna radiation attributes can be summarized in the next three parameters: pointing direction $\theta_{m}$, main lobe $3 \mathrm{~dB}$-beamwidth $\Delta \theta$, and main lobe to sidelobe level (SLL).

Different technologies have been applied to conceive radiators which are able to control these characteristics; wire antennas, waveguide apertures, printed circuit antennas, surfacewave antennas and leaky-wave antennas (LWAs) [1]. It is well known that the illumination of the near fields determines the far field characteristics. In this way, the beamwidth $\Delta \theta$ is inversely related to the effective antenna surface which is illuminated. Also, the pointing direction $\theta_{m}$ can be adjusted by applying a linear phase illumination, and the SLL depends on the shape of the illumination function [1]. LWAs are a type of travelling wave antennas which show some interesting features in order to control the radiation patterns [2]. The excited leaky-mode complex

This work was

supported in part by Spanish national projects ESP2001-4546-PE, TEC200404313-C02-02-TCM and in part by regional Séneca project 2002 PB/4/FS/02.

J. L. Gómez-Tornero and A. Melcón are with the Departamento de Tecnologías de la Información y las Comunicaciones Universidad Politécnica de Cartagena, Cartagena 30202, Spain.

G. Goussetis is with the School of Engineering and Physical Sciences, HeriotWatt University, Edinburgh EH14 4AS, U.K.

A. P. Feresidis is with the Department of Electronic and Electrical Engineering, Loughborough University, Leicestershire LE11 3TU, U.K.

Color versions of Figs. 1-7 and 9-12 are available online at $h t t p: / / i e e x p l o r e$. ieee.org.

Digital Object Identifier 10.1109/TAP.2006.884298 propagation constant $(k=\beta-j \alpha)$ is straightforwardly related to the antenna radiation properties. The normalized phase constant $\left(\beta / k_{0}\right)$ determines the pointing direction $\left(\theta_{m}\right)$, while the normalized leakage constant $\left(\alpha / k_{0}\right)$, together with the antenna length $\left(L_{A}\right)$ establish the effective radiating length, and therefore the main lobe beamwidth $(\Delta \theta)$. On the other hand, the SLL can be varied by tapering the leaky-mode leakage rate along the antenna length. Therefore, by controlling the leaky-mode complex propagation constant, one can determine the radiation characteristics of a LWA.

The procedure to taper the antenna dimensions in order to obtain a given radiation diagram depends on each particular type of LWA. Several hollow waveguide designs have been proposed to conceive original tapered LWAs, as the groove guide with an asymmetric strip [3], [4], the offset groove guide [5], the stub loaded rectangular waveguide [6], the slitted asymmetric ridge waveguide [7] and the stepped rectangular waveguide [8]. LWAs using the non-radiative dielectric guide (NRD) technology [9] have also been proposed. By using a dielectric filled guide, better frequency scanning sensibility is obtained, when compared to the hollow counterpart antennas [2]. Also, the NRD technology is very suitable for millimeter waveband applications [9]. Several NRD structures have been studied to transform the original bounded mode into a radiative leaky-mode [10]-[15].

All the previously proposed LWA make use of a waveguide in which some kind of asymmetric modification is added to induce radiation. Moreover, the robust waveguide geometry must be modified to control the leakage rate and taper the illumination. This introduces complicated and expensive mechanization processes to design a tapered antenna. A hybrid dielectric-waveguide printed-circuit (HDWPC) technology was originally proposed by Lampariello and Oliner in [16]. In that work, the main leaky-mode complex propagation constant was obtained using a transverse resonance technique. It was shown that a structure as the one presented in Fig. 1 can induce and control the leakage rate, by only modifying the printed-circuit dimensions. This technology was studied with more detail in [17] using a leaky-mode analysis technique based on the method of moment, developed in [18]. A quite similar technology was used in a paper by Whetten and Balanis [19], where a meandered long slot was applied to the broad wall of a rectangular waveguide to induce and control the radiation from the main mode. However, the LWA antenna proposed in [19] is substantially different from the hybrid technology studied in this paper. In [19], a slot is cut on the broad wall of a hollow rectangular waveguide mounted 


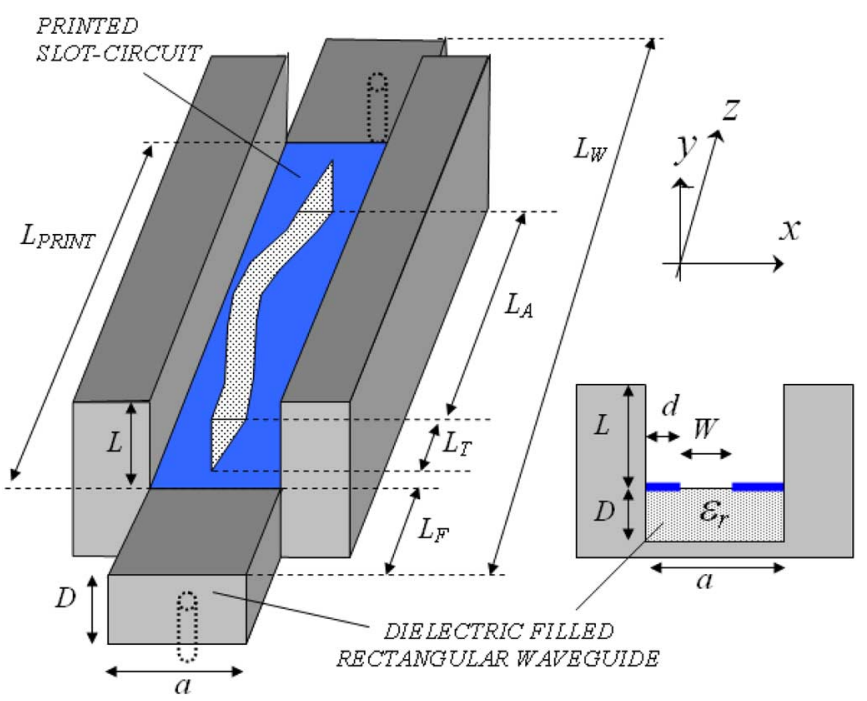

Fig. 1. Leaky-wave antenna in HDWPC technology.

in an infinite ground plane. The hybrid technology used in this paper makes use of a dielectric-filled rectangular waveguide, to which a slot printed-circuit is added in the top broad wall. This technique simplifies the manufacturing process of the structure. Moreover, the authors of [19] used a three-dimensional analysis technique, able to study the whole antenna structure. On the contrary, this paper is based on several two-dimensional analyses of the antenna cross-section, from which the evolution of the leaky-mode complex propagation constant can be obtained [17]. This approach leads to a straightforward and flexible design procedure for tapering the printed slot-circuit. On the opposite, it is suggested in [19] that, with the three-dimensional analysis, "the methodology for optimum tapering is not an easy task, and usually requires an iterative approach." Finally, no experimental results were shown in [19], but only simulated far field patterns were illustrated. In this paper, experimental results for HDWPC structures are shown for the first time. Measurements are compared with leaky-wave modal theoretical results in order to illustrate the ability to control the radiation properties of the perturbed $\mathrm{TE}_{10}$ leaky-mode by only modifying the printed circuit. Several designs are manufactured in this technology, showing experimentally the ability to control its radiation properties (SLL, pointing direction, beamwidth). Section II introduces the manufactured prototype, together with the description of the constituent parts of the antenna and its main geometrical dimensions. Section III presents results for uniform-slot LWAs, illustrating the accuracy between theory and experiments for the frequency beam-shifting response of the antenna, and for the radiation patterns. Section IV shows the ability to reduce the SLL by properly tapering the printed slot-circuit. Finally, it is shown in Section V how the pointing angle of the antenna can also be tuned by only modifying the printed-circuit.

\section{Hybrid DiELECTRIC-WAVEGUIDE PRINTED-CIRCUIT TECHNOLOGY}

Fig. 1 shows the structure considered in this work, together with the main geometrical parameters involved in the study.

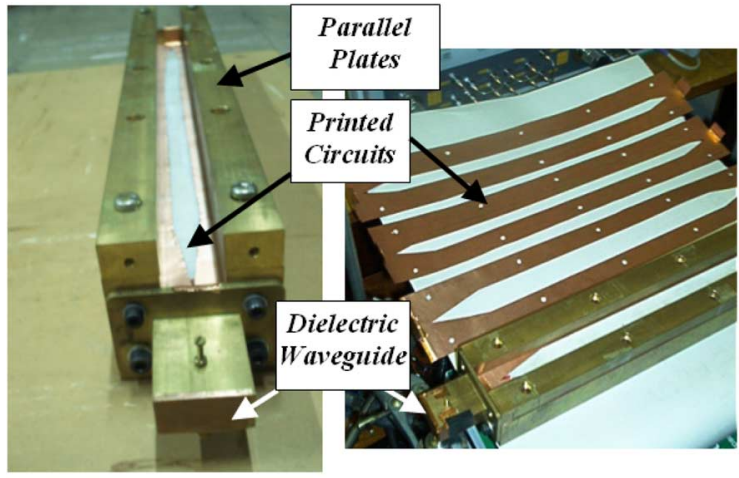

a)

b)

Fig. 2. Photographs of manufactured structure in hybrid technology. (a) Complete structure. (b) Detail of mountable printed slot-circuits.

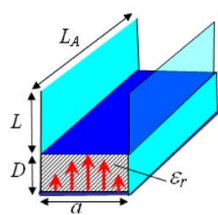

a)

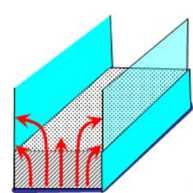

b)

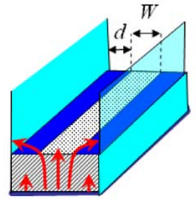

c)

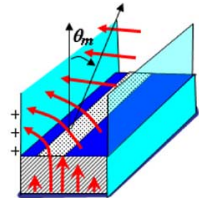

d)
Fig. 3. Perturbed $\mathrm{TE}_{10}$ mode in several structures related to the HDWPC technology: (a) Closed dielectric rectangular waveguide (DRWG). (b) NRD. (c) Centred slot HDWPC guide. (d) Asymmetric slot HDWPC guide.

A dielectric-filled (relative permittivity $\varepsilon_{r}$ ) rectangular waveguide of width $a$ and height $D$ is conveniently fed to excite the main $\mathrm{TE}_{10}$ mode. Except for the feeding sections of length $L_{F}$, the top wall of the waveguide is open along a length equal to $L_{\text {PRINT }}$, where a printed circuit will be added in the dielectric-air interface (see Fig. 1). Two metallic parallel plates (of height $L$ and separated a width $a$ ), are used along all the printed circuit length to form lateral walls. As it will be explained, a printed slot-circuit will be used to induce radiation from the originally bounded $\mathrm{TE}_{10}$ mode of the dielectric guide. In order to control the radiation properties of the LWA, the position $d$ and the width $W$ of the slot-circuit will be modulated along the antenna length, $L_{A}$, as it is illustrated in Fig. 1. Triangular transitions of length $L_{T}$ will be used at both ends of the antenna to reduce the discontinuity effects between the completely closed waveguide and the printed slot-circuit (see Fig. 1), improving the input matching and avoiding diffraction.

The structure is basically composed on three parts: a rectangular dielectric-waveguide, a printed slot-circuit, and two parallel plates [see Fig. 2(a) and (b)]. The dielectric guide parameters (width $a$, height $D$ and dielectric permittivity $\varepsilon_{r}$, see Fig. 1) will determine the frequency band of operation of the perturbed $\mathrm{TE}_{10}$ mode. The slot printed-circuit dimensions (position $d$ and width $W$, see Fig. 1) will make the originally bounded $\mathrm{TE}_{10}$ mode of the dielectric guide to become leaky. Finally, the height of the parallel plates ( $L$, see Fig. 1), will avoid spurious radiation from evanescent surface-wave fields, improving the horizontal polarization purity [20].

This working mechanism is based on the asymmetrical location of the printed circuit [17]. Fig. 3 shows several waveguides related to the HDWPC technology, together with the elec- 
tric field lines of the main mode, which is basically a perturbation of the $\mathrm{TE}_{10}$ mode of the dielectric rectangular waveguide (DRWG) [see Fig. 3(a)]. This DRWG structure can be considered as a limiting case of the HDWPC technology, since no slot is used. Another limiting case is the non radiative dielectric guide (NRD) [see Fig. 3(b)], in which the slot width is equal to the waveguide width $(W=a)$. Any laterally shielded symmetric-slot structure can propagate a non-radiative perturbed $\mathrm{TE}_{10}$ mode [Fig. 3(b) and (c)]. The asymmetric counterpart Fig. 3(d) will induce radiation by means of a leaky-wave perturbed $\mathrm{TE}_{10}$ mode.

As it has been commented, the main advantage of the HDWPC technology when compared to other previously presented LWAs, is that the tapered printed-circuit is responsible for controlling the leaky-mode complex propagation constant, and therefore the radiation properties of the structure. In this way, one can easily design and manufacture the printed-circuits by using common photolithographic processes see Fig. 2(b). Then, the printed circuits can be easily inserted into the top interface of the host dielectric guide. There is no need to taper the waveguide dimensions themselves, avoiding complex and expensive mechanization processes. This design is also much more flexible than previous LWA based completely on waveguide technology, since one can use the same host guide and interchange different printed circuits to obtain different radiation specifications. To experimentally prove the leaky-wave characteristics of this structure, a prototype has been constructed using an $X$-band waveguide ( $a=22.86 \mathrm{~mm}$, $D=10.16 \mathrm{~mm}, L_{W}=480 \mathrm{~mm}$, with $L_{F}=40 \mathrm{~mm}$, see Fig. 1) filled with PTFE (Teflon, $\varepsilon_{r}=2.2, \tan \delta=0.005$ ), and using parallel-plates of height $L=30 \mathrm{~mm}$.

\section{UNIFORM SLOT LEAKY-WAVE ANTENNA}

Using a modal analysis technique based on the method of moments, specifically developed for this type of HDWPC structures [17], [18], the evolution of the complex propagation constant of the perturbed $\mathrm{TE}_{10}$ leaky mode can be obtained for any longitudinal position of the antenna ( $z$-axis, see Fig. 1)

$$
k(z)=\beta(z)-j \alpha(z) \quad\left(m^{-1}\right)
$$

where $\beta$ is the phase constant and $\alpha$ is the attenuation constant. The attenuation constant can be split in three different attenuation rates: reactive attenuation rate, dielectric losses attenuation rate, and leakage (radiation) losses attenuation rate

$$
\alpha(z)=\alpha^{\mathrm{REACT}}(z)+\alpha^{\mathrm{RAD}}(z)+\alpha^{\mathrm{DIE}}(n e p / m)
$$

where $\alpha^{\mathrm{DIE}}$ has been assumed to be independent of the " $z$ " coordinate. Once the total attenuation constant is obtained, and assuming perfect matching, the insertion losses can be computed from the leaky-mode analysis as

$$
I L=S_{21}(d B)=20 \cdot \log _{10}\left\{e^{-\int_{z=0}^{z=L_{W}} \alpha(z) \cdot d z}\right\}
$$

The near field complex function along the antenna aperture axis ( $z$-axis, see Fig. 1) can be obtained from the next expression [2]

$$
\begin{array}{r}
M(z)=\sqrt{\alpha^{\mathrm{RAD}}(z) \cdot e^{-2 \cdot \int_{\tau=0}^{\tau=z}\left(\alpha^{\mathrm{RAD}}(\tau)+\alpha^{\mathrm{DIE}}(\tau)\right) \cdot d \tau}} \\
\cdot e^{-j \beta(z) \cdot z} .
\end{array}
$$

Finally, taking the Fourier transform of (4), the far-field radiation pattern in the H-plane (Y-Z plane in Fig. 1) can be straightforwardly derived as

$R(\theta)=20 \cdot \log \left\{\int_{z=0}^{z=L_{W}} M(z) \cdot e^{j k_{0} z \sin (\theta)} \cdot d z\right\}$

where $\theta$ is the elevation or pointing angle [see Fig. 3(d)].

In the case of a uniform slot-circuit, $\beta, \alpha^{\mathrm{RAD}}$ and $\alpha^{\mathrm{DIE}}$ are constant along the antenna length, therefore not depending on the longitudinal position " $z$." As illustrated in Fig. 3(d), the asymmetry introduced by the printed-slot makes the originally bounded perturbed $\mathrm{TE}_{10}$ mode of the NRD guide to transform into a radiating leaky-mode. To demonstrate this theory, four different uniform slot-circuits have been constructed. All of them have a width $W=12 \mathrm{~mm}$, but each one has a different slot-offset $d$ (see Fig. 1). The first one is a centered slot of width $W=12 \mathrm{~mm}$ and $d=5.43 \mathrm{~mm}$, in which no radiation occurs see Fig. 3(c). The three next slot-circuits are shifted to one of the side walls to break the symmetry see Fig. 3(d), having $d=5 \mathrm{~mm}, d=4.5 \mathrm{~mm}$ and $d=3 \mathrm{~mm}$ respectively. Fig. 4(a) shows the measured $\mathrm{S}_{21}$, demonstrating that the insertion losses increases for stronger asymmetry (as $d$ is smaller), due to the increase of the leakage rate, $\alpha^{\mathrm{RAD}}$.

Fig. 4(b) shows the numerical results for the perturbed $\mathrm{TE}_{10}$ mode complex propagation constant for the centered $(W=$ $12 \mathrm{~mm} d=5.43 \mathrm{~mm}$ ) and for two non-centered slot-circuits ( $W=12 \mathrm{~mm}, d=5 \mathrm{~mm}$, and $d=3 \mathrm{~mm}$ ). The cutoff frequency is approximately the same in the three circuits (around $5.2 \mathrm{GHz}$ ), and it is meanly determined by the waveguide width " $a$ " and relative permittivity " $\varepsilon_{r}$ " (as it is usual in a perturbed $\mathrm{TE}_{10}$ mode). Above cutoff, the normalized attenuation constant $\left(\alpha / k_{0}\right)$ is higher as the circuit asymmetry increases (as " $d$ " decreases), due to increased radiation losses. With the computed complex propagation constant, the insertion losses are calculated following the modal approach (3), and they are plotted with symbols in Fig. 4(a). Good agreement is observed between modal analysis results and experiments, therefore validating the modal analysis approach.

In order to better illustrate the asymmetry radiation mechanism, Fig. 5 shows the normalized phase $\left(\beta / k_{0}\right)$ and leakage constant $\left(\alpha^{\mathrm{RAD}} / k_{0}\right)$ obtained from the perturbed $\mathrm{TE}_{10}$ mode simulations (continuous line), as a function of the slot position " $d$ " for a constant width $W=12 \mathrm{~mm}$. The analysis frequency is $f=6 \mathrm{GHz}$. The measured leakage rate $\alpha^{\mathrm{RAD}}$ can be computed from (2) and (3) (once the constant $\alpha^{\mathrm{DIE}}$ is known from the material losses), and it is plotted with circles for the four manufactured circuits $(d=5.43 \mathrm{~mm}, d=5 \mathrm{~mm}, d=4.5 \mathrm{~mm}$, and $d=3 \mathrm{~mm}$ ). As it is sketched in the drawings of Fig. 5, as the slot-circuit is more uncentered, the launched horizontal 


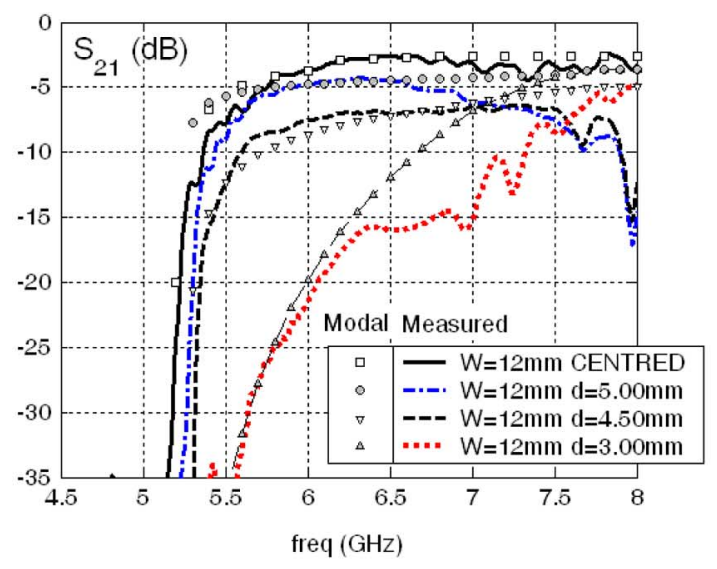

a)
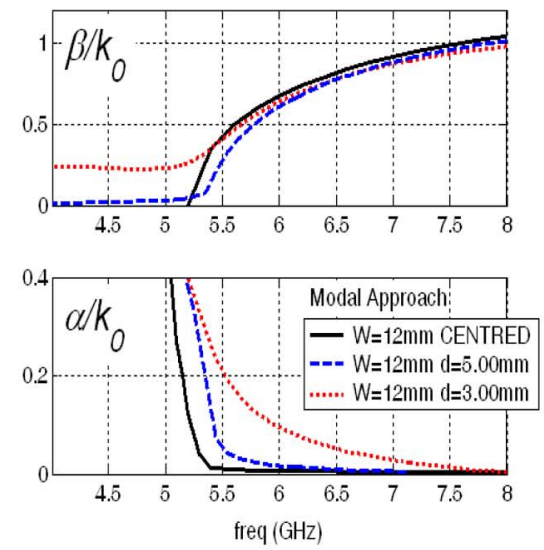

b)

Fig. 4. (a) Simulated and measured $S_{21}$ for non-centered slots of width $W=$ $12 \mathrm{~mm}$ and different position $d$. (b) Simulated perturbed $\mathrm{TE}_{10}$ mode complex propagation constant.
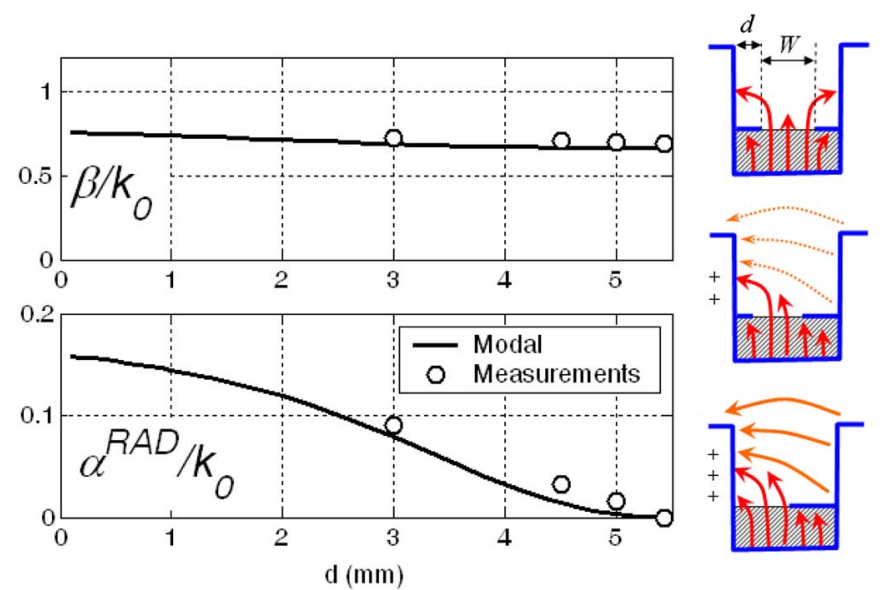

Fig. 5. Simulated and measured perturbed $\mathrm{TE}_{10}$ mode normalized complex propagation constant as a function of $d$ for slot-circuits of constant width $W=$ $12 \mathrm{~mm}$ at $6 \mathrm{GHz}$.

leaky-wave amplitude is stronger due to the higher difference between the charges induced in the two side walls [17]. In this way, the leakage rate can be controlled from zero (centered circuit) to a maximum value, by just modifying the slot offset.

For a uniform LWA, (4) describes a linear-phase exponentially-decaying-amplitude complex illumination function. The

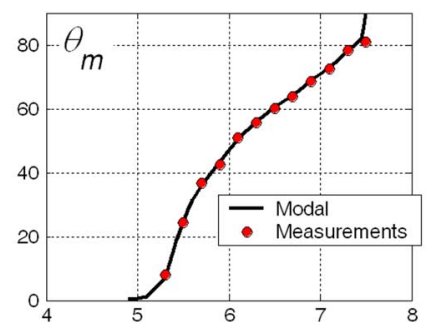

a)

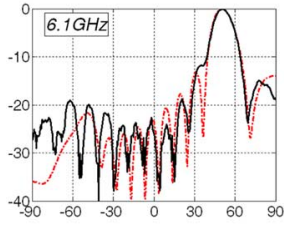

b)

req $(\mathrm{GHz})$

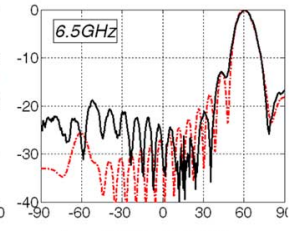

b)

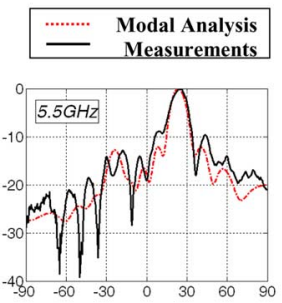

b) $\theta$ (degrees)
Fig. 6. Simulated and measured radiation properties of asymmetric slot circuit $W=12 \mathrm{~mm} d=5 \mathrm{~mm}, L_{A}=270 \mathrm{~mm}\left(L_{A}=5.4 \lambda_{0}\right.$ at $\left.6 \mathrm{GHz}\right)$.

theoretical radiation pattern (5) has a maximum at an elevation angle, $\theta_{m}$, given by [2]

$$
\theta_{m}=\arcsin \frac{\beta}{k_{0}} .
$$

Using (6), the phase constant is extracted from the measured pointing direction of the LWAs. In Fig. 5, comparisons between theory and measurements for $\beta / k_{0}$ are also plotted, showing again very good agreement.

It must be noticed that the perturbed $\mathrm{TE}_{10}$ mode frequency dispersion curve for $\beta$ [see Fig. 4(b)] can be translated into a frequency beam scanning curve, according to (6). In order to obtain a real $\theta_{m}$ pointing angle, $\beta / k_{0}$ must be lower than unity [2]. Following this theory and according to the $\beta / k_{0}$ curve shown in Fig. 4(b), the uncentered slot-circuits provide radiation from $5.2 \mathrm{GHz}$ (cutoff frequency) to $7.5 \mathrm{GHz}$ (from where the condition $\beta / k_{0}<1$ is no more satisfied). Beyond $7.5 \mathrm{GHz}$, the perturbed $\mathrm{TE}_{10}$ mode becomes a slow wave, also called bounded wave [2]. Fig. 6(a) shows the theoretical frequency beam scanning curve obtained for the slot-circuit with $W=12 \mathrm{~mm}$ and $d=5 \mathrm{~mm}$. Measured results are also plotted with circles, showing excellent agreement. Also in Fig. 6(b), the normalized radiation diagrams are shown for several frequencies. The radiation patterns obtained with the modal approach are also presented. The SLL corresponding to an exponential amplitude illumination is $13 \mathrm{~dB}$. Good agreement with measurements can be observed for the beamwidth and the SLL, for all frequencies.

\section{DESIGN OF TAPERED ANTENNAS FOR SIDELOBES REDUCTION}

In the previous sections, the asymmetry radiation mechanism has been illustrated by using $\mathrm{TE}_{10}$ mode complex propagation simulation results (modal approach), and experimental measurements. As a result, the function of each part of the proposed LWA in HDWPC technology has been studied. Once the host dielectric guide dimensions $\left(a, D\right.$ and $\left.\varepsilon_{r}\right)$ have been chosen to propagate a fast-wave perturbed $\mathrm{TE}_{10}$ mode in a 


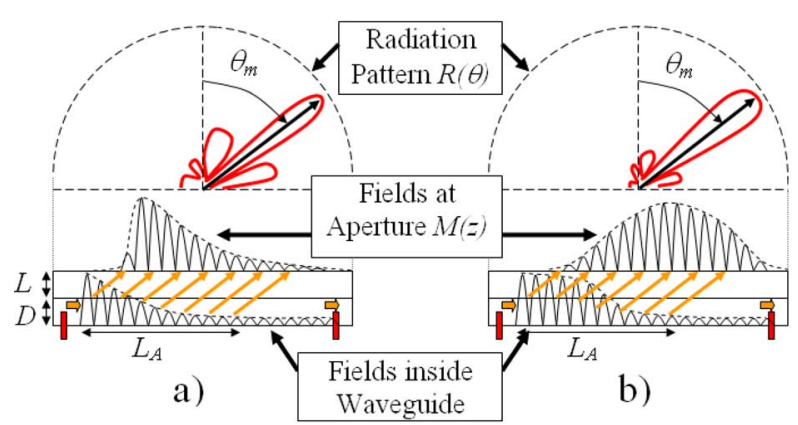

Fig. 7. Scheme illustrating (a) Nontapered LWA. (b) Tapered LWA.

given frequency band, the printed circuit dimensions ( $W$ and $d$ ) can be designed. The purpose of the printed circuit is to control the propagation and radiation properties of this perturbed $\mathrm{TE}_{10}$ leaky mode.

The control of $\beta / k_{0}$ and $\alpha / k_{0}$ is needed in order to perform a tapered LWA design [2]. Fig. 7 illustrates the difference between a non-tapered and a tapered LWA. A non-tapered LWA Fig. 7(a) is a uniform radiating structure which creates a linear-phase exponential-amplitude aperture illumination. The far field radiation pattern has a pointing angle $\theta_{m}$ defined by the normalized leaky-mode phase constant $\left(\beta / k_{0}\right.$, see (6), and the SLL are theoretically around $13 \mathrm{~dB}$, as it was shown in the measured results of the last section (see Fig. 6). In order to increase the SLL, a tapered-amplitude illumination [such as a cosine aperture illumination function, see Fig. 7(b)] must be synthesized along the antenna aperture length. For this purpose, the designer must be able to control the leaky-mode radiation rate $\left(\alpha^{\mathrm{RAD}} / k_{0}\right)$ from zero to a maximum value. Moreover, in order not to spoil the pointing direction $\theta_{m}$ of the LWA [17], the leaky-mode phase constant $\left(\beta / k_{0}\right)$ must be kept unchanged along all the antenna length, as illustrated in Fig. 7(b).

Following these ideas, the position of the slot " $d$ " can be varied to control the normalized leakage rate $\alpha^{\mathrm{RAD}}$, by means of the asymmetry radiation mechanism. However, it can be seen in Fig. 5 that the phase constant $\beta$ changes when " $d$ " is varied. In order to vary $\alpha / k_{0}$ without affecting $\beta / k_{0}$ (without modifying $\theta_{m}$ ), the slot position and width (" $W$ " and " $d$ ") must be simultaneously modified along the antenna length [17]. The tapered design procedure is sketched in Fig. 8. First, the illumination amplitude function along the antenna aperture $(M(z))$ is chosen. The equivalence between the near field function $M(z)$ and the radiation pattern is well known [1], see (5). The variation of the leaky-mode leakage rate along the antenna length $\left(\alpha^{\mathrm{RAD}}(z)\right)$ can be computed from the illumination function to be synthesized $M(z)$, according to the next expression ( $\eta^{\mathrm{RAD}}$ is the radiation efficiency) [2]

$$
\alpha^{\mathrm{RAD}}(z)=\frac{1}{2} \frac{M(z)}{\frac{1}{\eta^{\mathrm{RAD}}} \int_{z=0}^{L_{A}} M(z) \partial z-\int_{z=0}^{z} M(z) \partial z} .
$$

Fig. 8(a) shows the variation of the normalized leakage rate along the tapered printed-circuit slot length [obtained from (7)], needed to synthesize a cosine illumination function $M(z)$ (with $\eta^{\mathrm{RAD}}=90 \%$ ). On the other hand, the leaky mode phase constant must be kept unchanged along all the antenna length, so

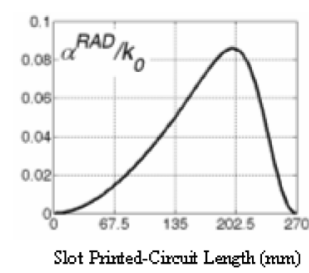

a)

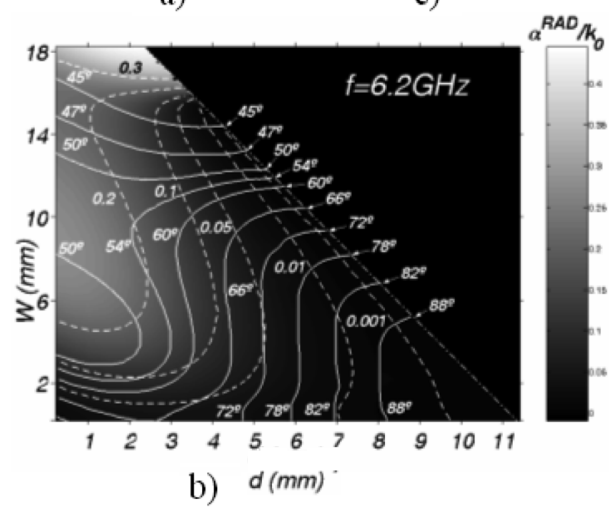

Fig. 8. Tapered LWA design procedure.

that all sections of the LWA radiate at the same pointing angle $\theta_{m}$ [see (6)].

In order to vary $\alpha^{\mathrm{RAD}} / k_{0}$ while keeping $\theta_{m}$ constant, the printed slot-circuit dimensions (width " $W$ " and position " $d$ ") must be adjusted for each longitudinal position of the antenna, " $z$." For this purpose, the leaky-mode contour plots shown in Fig. 8(b) are used. These curves were obtained from the $\mathrm{TE}_{10}$ modal analysis at $6.2 \mathrm{GHz}$, by computing the leaky-mode complex propagation constant for $L=20 \mathrm{~mm}, a=22.86 \mathrm{~mm}$, $D=10.16 \mathrm{~mm}$, and $\varepsilon_{r}=2$, and simultaneously varying $W$ and $d$. The continuous lines define the set of dimensions (" $W$ " and " $d$ ") which have a common pointing angle $\theta_{m}$, while the dashed lines are for a constant leakage rate $\alpha^{\mathrm{RAD}} / k_{0}$. If we move along a constant pointing angle line we can obtain the set of dimensions " $W$ " and " $d$ " for each longitudinal position " $z$ " [see Fig. 8(c)], which allow to vary $\alpha^{\mathrm{RAD}}$ in a specified fashion while keeping $\theta_{m}$ unchanged. Finally, the printed circuit mask can be manufactured with photolithographic processes using these dimensions, as shown in Fig. 8(d).

In order to see the improvement obtained by tapering the printed slot-circuit, Fig. 9 shows measured radiation patterns for a non-tapered [uniform circuit, Fig. 9(a)] and a cosine-tapered LWA in HDWPC technology [Fig. 9(b)]. Both antennas present a pointing angle $\theta_{m}=54^{\circ}$ for $6.2 \mathrm{GHz}$, but a considerable improvement in the SLL (around $10 \mathrm{~dB}$ ) is observed for the tapered design. The main lobe is wider for the tapered antenna, as expected from the shorter effective length corresponding to the tapered radiating aperture. However, it must be noticed that the beamwidth can be controlled by adjusting the physical length of the antenna, $L_{A}$. The simulated radiation patterns obtained with the modal approach are also plotted. The agreement observed validates the leaky mode analysis and the design procedure.

Fig. 10(a) shows the measured radiation pattern of the designed cosine tapered LWA for different frequencies. It can be seen that the sidelobes reduction is maintained for different 


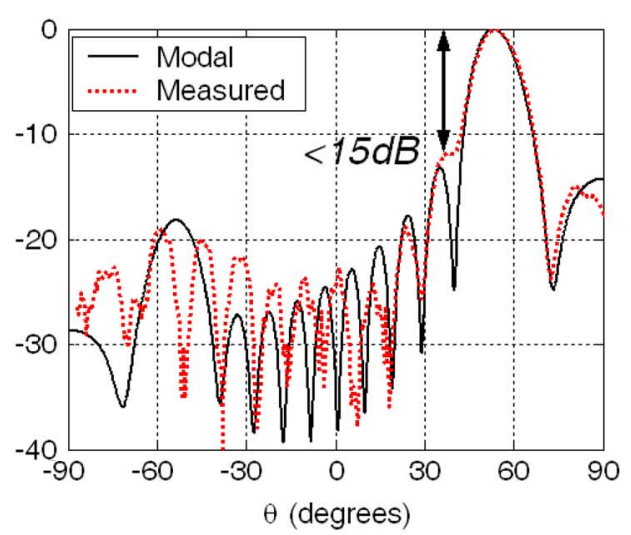

a)

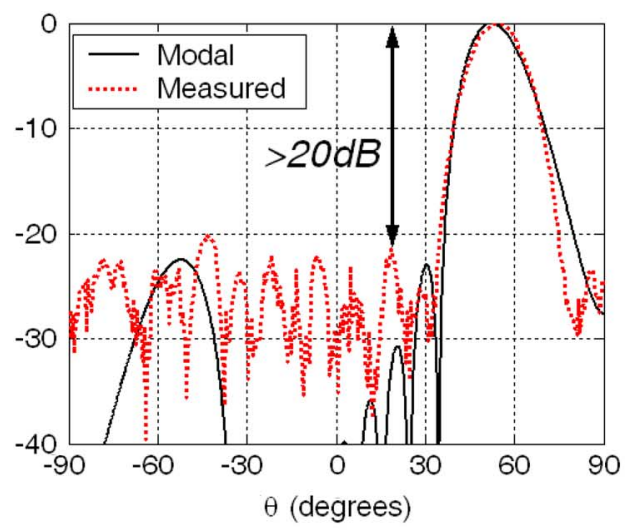

b)

Fig. 9. Measured and simulated radiation patterns at $6.2 \mathrm{GHz}$ (a) Non-tapered printed-circuit. (b) Tapered printed-circuit.

scanning angles. The scattering parameters of the designed tapered LWA are plotted in Fig. 10(b). Measurements are in good agreement with both HFSS three-dimensional simulations, and also with the results of $S_{21}$ obtained from the leaky mode approach analysis (3).

\section{Controlling the Pointing Direction}

In the previous section, it has been demonstrated that a tapered slot printed-circuit can be added at the top of an NRD guide in order to modulate the leakage rate of the perturbed $\mathrm{TE}_{10}$ leaky-mode. In this way, it can be obtained a radiation pattern pointing at a given angle $\theta_{m}$, with lower SLL than in the case of a uniform antenna. The pointing angle $\theta_{m}$ is defined by the leaky-mode normalized phase constant, $\beta / k_{0}$, which primarily depends on the dielectric waveguide dimensions (" $a$," " $\varepsilon_{r}$," and " $D$ ") and the frequency. However, the printed circuit can also be adjusted to tune the pointing direction of the antenna over a range of angles for a fixed frequency.

As it can be seen in the contour leaky-mode dispersion graph shown in Fig. 8(b), the pointing angle $\theta_{m}$ can be varied in a range of 20 degrees by modifying the printed circuit dimensions " $W$ " and " $d . "$ Basically, by choosing a wider or a narrower slot design, the cutoff frequency of the perturbed $\mathrm{TE}_{10}$ mode can be shifted. The cutoff frequency increases as the slot width " $W$ " is larger, due to the decrease of the effective dielectric constant of

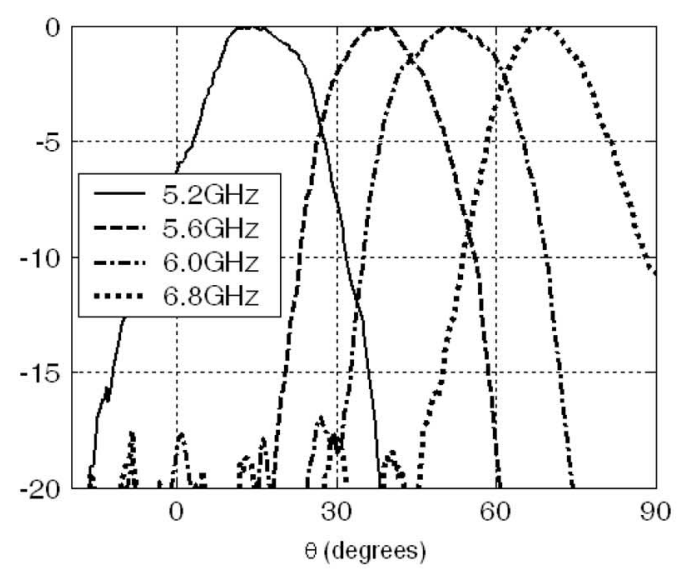

a)

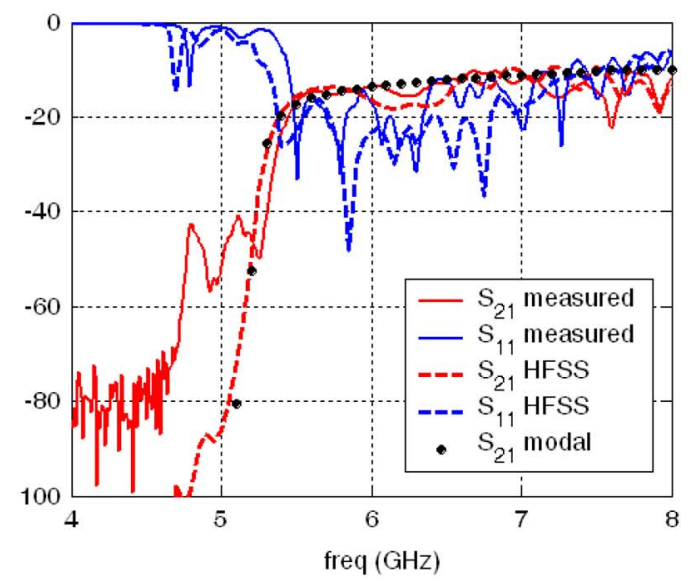

b)

Fig. 10. (a) Measured radiation patterns for tapered LWA at different frequencies. (b) Measured and simulated S parameters for tapered LWA in HDWPC technology.

TABLE I

DiMENSIONS OF THE TAPERED PRINTED-CIRCUITS FOR THE THREE ANTENNAS

\begin{tabular}{|c|c|c|c|c|c|c|}
\hline & \multicolumn{2}{|c|}{$\begin{array}{c}\text { ANTENNA 1 } \\
\theta_{m}=66^{\circ} @ 6,2 \mathrm{GHz}\end{array}$} & \multicolumn{2}{|c|}{$\begin{array}{c}\text { ANTENNA 2 } \\
\theta_{m}=54^{\circ} @ 6,2 \mathrm{GHz}\end{array}$} & \multicolumn{2}{|c|}{$\begin{array}{c}\text { ANTENNA 3 } \\
\theta_{m}=47^{\circ} @ 6,2 \mathrm{GH}\end{array}$} \\
\hline $\mathrm{z}(\mathrm{mm})$ & $\mathrm{d}(\mathrm{mm})$ & $W(\mathrm{~mm})$ & $\mathrm{d}(\mathrm{m} m)$ & $W(\mathrm{~mm})$ & $\mathrm{d}(\mathrm{mm})$ & $\mathrm{W}(\mathrm{mm})$ \\
\hline 0,00 & 6,29 & 9,88 & 5,22 & 12,29 & 4,24 & 14,20 \\
\hline 27,0 & & 9,88 & 4,9 & 12 & 4,20 & 14,20 \\
\hline 54,0 & 5,88 & 9,80 & 4,6 & 12 & 4,0 & 14,20 \\
\hline 81,00 & 5,61 & 9,67 & 4,38 & 12,20 & 3,90 & 14,20 \\
\hline 108,00 & 5 & 9,47 & 4,12 & 12,15 & 3,74 & 14,20 \\
\hline 135,00 & 5,21 & 9,26 & 3,91 & 12,09 & 3,62 & 14,20 \\
\hline 162,00 & 12 & 9,12 & 3,76 & 12,05 & 3,55 & 14,20 \\
\hline & 5,1 & $y_{1} 1$ & 3,7 & 12 & 3,58 & 14,20 \\
\hline & 5 & 9,46 & 4,0 & 12,12 & 3,73 & 14,20 \\
\hline 243,00 & 5,80 & 9,80 & 4,59 & 12,24 & 4,05 & 14,20 \\
\hline 270,00 & 6,29 & 9,88 & 5,22 & 12,29 & 4,24 & 14,20 \\
\hline
\end{tabular}

the perturbed $\mathrm{TE}_{10}$ mode. In this way, one can choose a wider or narrower slot to tune the pointing angle. Table I shows the dimensions of three cosine-tapered LWAs, designed using the contour curves shown in Fig. 11 to synthesize beams pointing at $\theta_{m}=47^{\circ}, 54^{\circ}$ and $66^{\circ}$, at a fixed frequency of $6.2 \mathrm{GHz}$. The slot width " $W$ " and the offset " $d$ " are varied along the antenna length $\left(L_{A}=270 \mathrm{~mm}\right)$, to obtain the desired leakage rate function $\left[\alpha^{\mathrm{RAD}}(z),(7)\right]$, while keeping $\theta_{m}$ constant to avoid phase aberrations. 


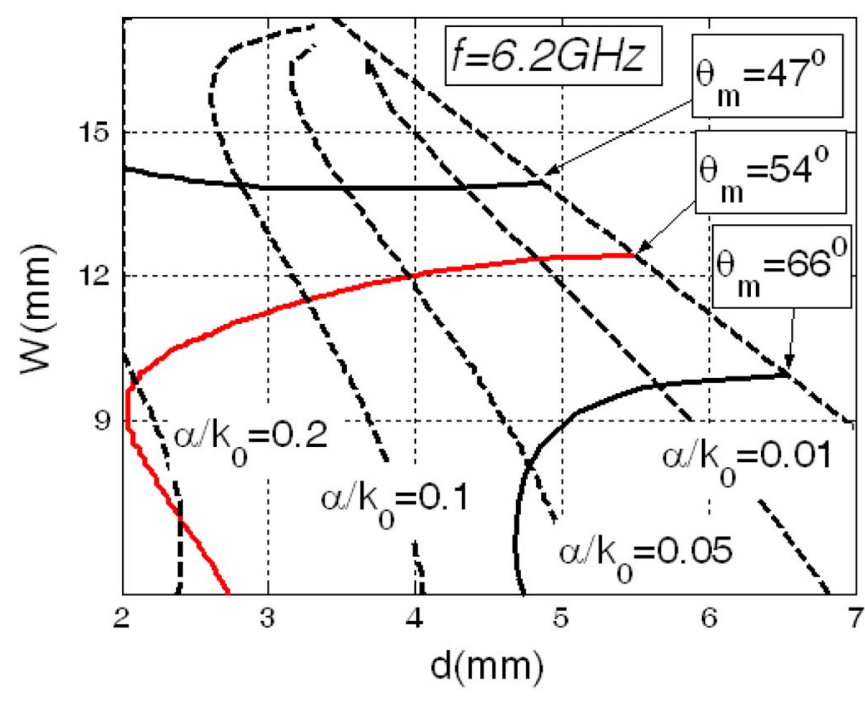

Fig. 11. Contour curves used to tune the pointing angle for tapered designs at $6.2 \mathrm{GHz}$.

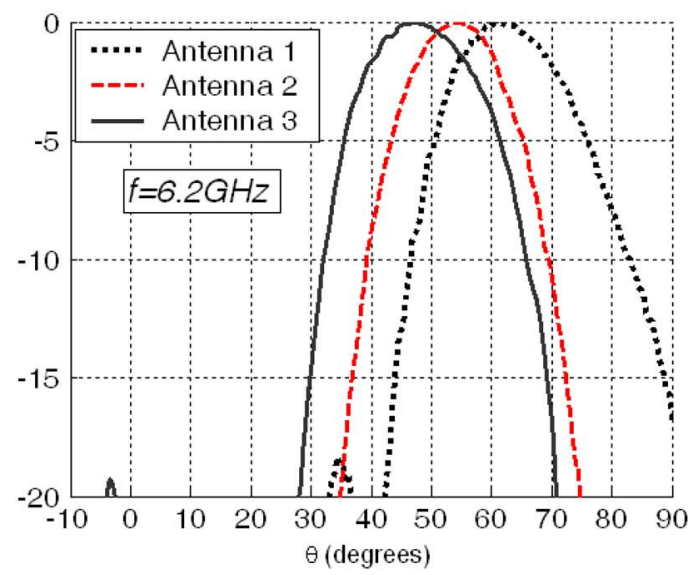

a)

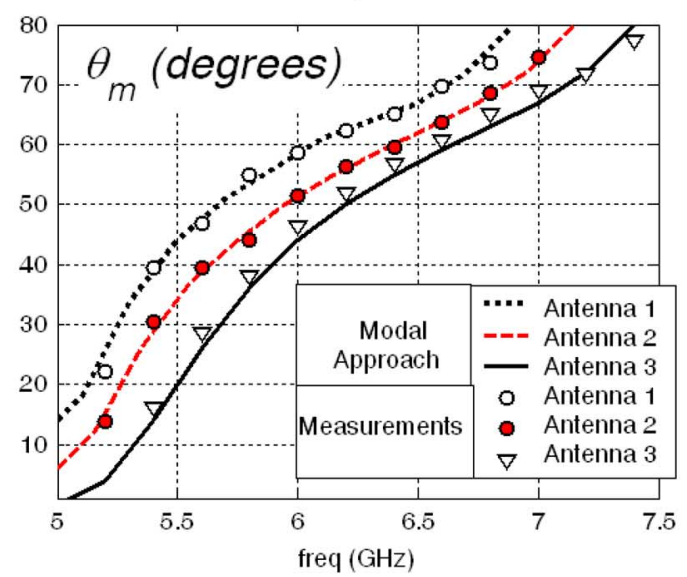

b)

Fig. 12. (a) Measured radiation pattern at $6.2 \mathrm{GHz}$ showing the tuning in $\theta_{\mathrm{m}}$. (b) Measured and simulated pointing direction $\theta_{\mathrm{m}}$ for the three tapered designs.

Fig. 12(a) shows the normalized radiation pattern of the three cosine-tapered antennas at the design frequency of $6.2 \mathrm{GHz}$, confirming the ability to tune the pointing angle while controlling the illumination to avoid high sidelobes levels. Fig. 12(b) shows the simulated and measured frequency beam scanning behavior for theses three antennas. Again, excellent agreement is obtained between modal approach and measurements. From the dispersion curves shown in Fig. 12(b), it is easily confirmed that the perturbed $\mathrm{TE}_{10}$ leaky-mode cutoff frequency is shifted to higher frequencies, when wider slots are used to induce radiation. In this way, the whole beam-scanning response is shifted, obtaining lower radiating angles for wider slots designs, as shown in Fig. 12(a).

\section{CONCLUSION}

In this paper, it has been demonstrated experimentally for the first time the ability to control the radiation properties of a leaky mode which propagates in a hybrid waveguide printed-circuit guide. This hybrid technology is based on a slot-circuit printed on the top side of a rectangular dielectric guide, and laterally shielded with parallel-plates. The function of each part of the structure to control the complex propagation constant of the perturbed $\mathrm{TE}_{10}$ leaky-mode has been described by simple and illustrative experiments performed on a prototype conceived to radiate in the $6 \mathrm{GHz}$ band. These leaky fields are excited using the asymmetry radiation mechanism, which is controlled by the position and dimensions of the slot printed-circuit. The main practical advantage with respect to other LWAs resides in the fact that the host waveguide must not be changed in order to obtain a given specification. The radiation properties of the antenna (pointing angle, beamwidth and sidelobes) can be controlled by only interchanging different printed-circuit masks, therefore avoiding complicated mechanization processes. In addition, a simple and straightforward design procedure based on the dispersion curves obtained from modal analysis has been used. Good agreement was observed between measurements and the leaky-wave modal approach, thus validating the proposed design procedure. This original hybrid dielectric-waveguide printed-circuit technology provides a good option to conceive simple LWA designs, in which the main radiation properties can be flexibly controlled.

\section{ACKNOWLEDGMENT}

The authors want to thank Epson-Ibérica Foundation, Vodafone Foundation, and the Spanish Institute of Telecommunication Engineers (COIT/AEIT) for their stimulating support. The authors also want to thank Prof. J. Yiannis, and C. Vardaxoglou from Loughborough University, U.K., for his helpful comments and for providing research facilities.

\section{REFERENCES}

[1] C. A. Balanis, Antenna Theory. New York: Wiley, 1982.

[2] A. A. Oliner, , R. C. Johnson, Ed., "Leaky-wave antennas," in Antenna Engineering Handbook, 3rd ed. New York: McGraw-Hill, 1993, ch. 10.

[3] P. Lampariello and A. A. Oliner, "A new leaky-wave antenna for millimeter waves using an asymmetric strip in groove guide-Part I: Theory," IEEE Trans. Antennas Propag., vol. 33, pp. 1285-1294, 1985.

[4] Z. Ma and E. Yamashita, "Leakage characteristics of groove guide having a conductor strip," IEEE Trans. Microw. Theory Tech., vol. 42, no. 10, pp. 1925-1931, 1994.

[5] P. Lampariello, F. Frezza, H. Shigesawa, M. Tsuji, and A. A. Oliner, "Guidance and leakage properties of offset groove guide," in 1987 IEEE MTT-S Int. Microwave Symp. Dig., pp. 731-734.

[6] P. Lampariello, F. Frezza, H. Shigesawa, M. Tsuji, and A. A. Oliner, “A versatile leaky-wave antenna based on stub-loaded rectangular waveguide-Part I: Theory," IEEE Trans. Antennas Propag., vol. 46, no. 7, pp. 1032-1041, Jul. 1998. 
[7] F. Frezza, M. Guglielmi, and P. Lampariello, "Millimeter-wave leakywave antennas based on slitted asymmetric ridge waveguides," Proc. Inst. Elect. Eng.: Microwaves, Antennas and Propagation, vol. 141, pt. H, pp. 175-180, Jun. 1994.

[8] C. Di Nallo, F. Frezza, A. Galli, and P. Lampariello, "Complete characterisation of leaky-wave antennas based on stepped rectangular waveguides," in Proc. 25th Eur. Microwave Conf., Bologna, Italy, Sep. 1995, pp. 1062-1067.

[9] T. Yoneyama and S. Nishida, "Nonradiative dielectric waveguide for millimeter-wave integrated circuits," IEEE Trans. Microw. Theory Tech., vol. 29, pp. 1188-1192, Nov. 1981.

[10] A. Sanchez and A. A. Oliner, "A new leaky waveguide for millimeter waves using nonradiative dielectric (NRD) waveguide-Part I: Accurate theory," IEEE Trans. Microw. Theory Tech., vol. 35, no. 8, pp. 737-747, Aug. 1987

[11] S. J. Xu, X.-Y. Zeng, K. Wu, and K.-M. Luk, "Characteristics and design consideration of leaky-wave NRD-guides for use as millimeter-wave antenna," IEEE Trans. Microw. Theory Tech., vol. 46, pp. 2450-2456, Dec. 1998

[12] A. A. Oliner, S. T. Peng, and K. M. Shang, "Leakage from a gap in NRD guide," in Proc. IEEE MTT Int. Microwave Symp. Dig., 1985, pp. 619-622.

[13] J. A. G. Malherbe, "A leaky-wave antenna in nonradiative dielectric waveguide," IEEE Trans. Antennas Propag., vol. 36, no. 9, pp. 1231-1235, Sep. 1988

[14] Z. Ma and E. Yamashita, "Wave leakage from groove NRD structures,' IEEE Microw. Guided Wave Lett., vol. 3, no. 6, pp. 170-172, Jun. 1993

[15] M. T. Lee, K. M. Luk, S. J. Xu, and E. K. N. Yung, "Leaky wave antenna based on image NRD guide with staircase shaped dielectric slab," Electron. Lett., vol. 36, no. 13, pp. 1102-1103, Jun. 2000.

[16] P. Lampariello and A. A. Oliner, "A novel phase array of printed-circuit leaky wave lines sources," in Proc. 17th Eur. Microwave Conf., Rome, Italy, 1987, pp. 555-560.

[17] J. L. Gómez, A. de la Torre, D. Cañete, M. Gugliemi, and A. A. Melcón, "Design of tapered leaky-wave antennas in hybrid waveguide-planar technology for millimeter waveband applications," IEEE Trans. Antennas Propag.: Special Issue on Antennas Propag. Applicat., vol. 53, no. 8, pt. I, pp. 2563-2577, Aug. 2005.

[18] J. L. Gómez and A. A. Melcón, "Non-orthogonality relations between complex-hybrid-modes: An application for the leaky-wave analysis of laterally-shielded top-open planar transmission lines," IEEE Trans. Microwave Theory Tech., vol. 52, no. 3, pp. 760-767, Mar. 2004.

[19] F. Whetten and C. A. Balanis, "Meandering long slot leaky-wave antennas," IEEE Trans. Antennas Propag., vol. 39, pp. 1553-1559, Nov. 1991.

[20] J. L. Gómez and A. A. Melcón, "Radiation analysis in the space domain of laterally-shielded planar transmission lines-Part II: Applications," Radio Sci., vol. 39, pp. 1-10, Jun. 2004, RS3006.

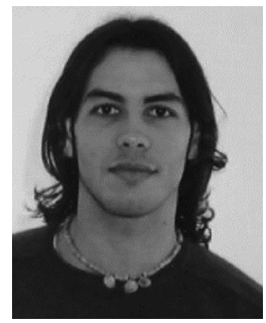

José Luis Gómez-Tornero (M'06) was born in Murcia, Spain, in 1977. He received the Telecommunications Engineer degree from the Polytechnic University of Valencia (UPV), Valencia, Spain, in 2001, and the (laurea cum laude) Ph.D. degree in telecommunication engineering from the Technical University of Cartagena (UPCT), Cartagena, Spain, in 2005 .

In 1999 he joined the Radiocommunications Department, UPV, as a research student, where he was involved in the development of analytical and numerical tools for the study and automated design of microwave filters in waveguide technology for space applications. In 2000, he joined the Radio Frequency Division, Industry Alcatel Espacio, Madrid, Spain, where he was involved with the development of microwave active circuits for telemetry, tracking and control (TTC) transponders implicated in many different spatial missions for the European Space Agency (ESA), National Aeronautics Space Administration (NASA) and other Space Agencies. In 2001, he joined the Technical University of Cartagena, Spain, as an Assistant Professor, where he is currently developing his teaching activities. From October 2005, he held the position of Vice Dean for Students and Lectures affairs in the Telecommunication Engineering Faculty at the Technical University of Cartagena. His scientific research is focused on the analysis and design of leaky-wave antennas for millimeter wave-band applications and the development of numerical methods for the analysis of novel passive radiating structures in planar and waveguide technologies. His scientific interests also include the study of active devices for microwave and millimeter wavebands, such as oscillators and active antennas.

Dr. Gómez Tornero received the second national award from the EPSONIbérica Foundation for the best Ph.D. project in the field of Technology of In- formation and Communications (TIC) in July 2004, and the Vodafone Foundation-COIT/AEIT award for the best Spanish Ph.D. thesis in the area of advanced mobile communications technologies, in June 2006.

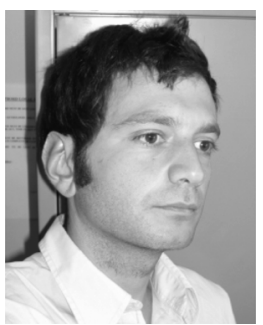

George Goussetis (M'01) was born in Athens, Greece, in 1976. He received the Electrical and Computer Engineering degree from the National Technical University of Athens, Greece, in 1998, and the B.Sc. degree in physics from University College, London, U.K. and the Ph.D. degree in the area of waveguide filters from the University of Westminster, London, U.K., both in 2002.

In 1998, he joined the Space Engineering Spa, Roma, Italy, as a Junior RF Engineer. In 1999, he joined the Wireless Communications Research Group, University of Westminster, as a Research Assistant. Currently, he is a Lecturer in the School of Engineering and Physical Sciences, Heriot-Watt University, Edinburgh, U.K. He has published in more than 70 peer reviewed journals and conference papers. His research interests include the modelling and design of microwave filters, properties of periodic structures as well numerical techniques for electromagnetics.

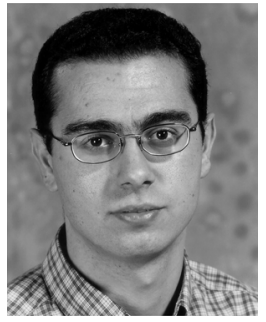

Alexandros P. Feresidis (S'98-M'01) was born in Thessaloniki, Greece, in 1975. He received the Physics degree from Aristotle University of Thessaloniki, Greece, the M.Sc.(Eng.) degree in radio communications and high frequency engineering from the University of Leeds, Leeds, U.K., and the $\mathrm{Ph} . \mathrm{D}$. in electronic and electrical engineering from Loughborough University, Leicestershire, U.K., in 1997, 1998, and 2002, respectively.

During the beginning of 2002, he was a Research Associate and was then appointed Lecturer in Wireless Communications in the Department of Electronic and Electrical Engineering, Loughborough University, where he is currently a Senior Lecturer in the same department. He has published more than 60 papers in peer reviewed international journals and conference proceedings. His research interests include analysis and design of artificial periodic metamaterials, electromagnetic bandgap (EBG) structures and frequency selective surfaces (FSS), high-gain array antennas, base station antennas, numerical techniques in electromagnetics and microwave circuits.

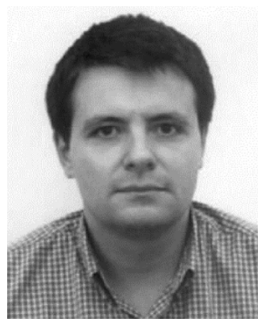

Alejandro Alvarez Melcón (M'98) was born in Madrid, Spain, in 1965. He received the Telecommunications Engineer degree from the Polytechnic University of Madrid (UPM), Madrid, Spain, in 1991 and the Ph.D. degree in electrical engineering from the Swiss Federal Institute of Technology, Lausanne, Switzerland, in 1998.

In 1988, he joined the Signal, Systems and Radiocommunications Department, UPM, as a Research Student, where he was involved in the design, testing, and measurement of broadband spiral antennas for electromagnetic measurements support (EMS) equipment. From 1991 to 1993, he joined the Radio Frequency Systems Division, European Space Agency (ESA/ESTEC), Noordwijk, The Netherlands, where he was involved in the development of analytical and numerical tools for the study of waveguide discontinuities, planar transmission lines, and microwave filters. From 1993 to 1995, he was with the Space Division, Industry Alcatel Espacio, Madrid, Spain, and he worked at the ESA, where he collaborated on several ESA/ESTEC contracts. From 1995 to 1999, he was with the Swiss Federal Institute of Technology, École Polytechnique Fédérale de Lausanne, Lausanne, Switzerland, where he worked in the field of microstrip antennas and printed circuits for space applications. In 2000, he joined the Technical University of Cartagena, Spain, where he is currently developing his teaching and research activities.

Dr. Alvarez Melcón received the Journée Internationales de Nice sur les Antennes (JINA) Best Paper Award for the best contribution to the JINA'98 International Symposium on Antennas, and the COIT/AEIT (Colegio Oficial de Ingenieros de Telecomunicación) award to the best $\mathrm{Ph}$.D. thesis in basic information and communication technologies. 